\title{
Improving Link Reliability of IEEE 802.15.4g SUN Networks with Adaptive Modulation Diversity
}

\author{
Ruan Delgado Gomes \\ Communications Systems and Information Processing Group \\ Federal Institute of Paraíba (IFPB) \\ Campina Grande, Brazil \\ \{ruan.gomes\}@ifpb.edu.br
}

\author{
Pere Tuset-Peiró, Xavier Vilajosana \\ Wireless Networks Laboratory \\ Internet Interdisciplinary Institute (IN3) \\ Universitat Oberta de Catalunya (UOC) \\ Barcelona, Spain \\ \{peretuset,xvilajosana\}@uoc.edu
}

\begin{abstract}
Adaptive mechanisms, such as channel hopping and packet replication, are used in low-power wireless networks to deal with the spatial and temporal variations in the link quality, and meet the reliability requirements of industrial applications (i.e., PDR $>99 \%$ ). However, the benefits of such mechanisms are limited and may have a large impact on end-to-end latency and energy consumption. Hence, in this paper we propose using adaptive modulation diversity, which allows to dynamically select different modulations, to improve link reliability. We present three adaptive modulation diversity selection strategies and validate them using the data derived from a real-world deployment using the IEEE 802.15.4g SUN modulations (i.e., SUN-FSK, SUNOQPSK and SUN-ODFM) in an industrial environment. The results show that by using adaptive modulation diversity it is possible to improve link reliability regardless of node conditions.
\end{abstract}

Index Terms-Industrial Wireless Sensor Networks, IEEE 802.15.4g, Smart Utility Networks, Link Reliability, Adaptive Techniques, Modulation Diversity.

\section{INTRODUCTION}

The wireless channel is inherently unreliable due to propagation and interference effects. In addition, temporal and spatial variations occur at both short and long term intervals, leading to unreliable operation [1]. To mitigate these effects, the standards for low-power wireless communications in the industrial domain, typically based on IEEE 802.15.4 [2] (i.e., WirelessHART, ISA100.11a and 6TiSCH [3]), use channel hopping at the data-link layer. Moreover, these networks take advantage of packet replication [4] at the network layer to further increase reliability. However, such mechanisms may be insufficient to meet the dependability requirements of certain industrial applications (i.e., PDR $>99 \%$ ), and have an impact on end-to-end latency and energy consumption.

Recently, the IEEE 802.15.4 standard [5] has incorporated the IEEE 802.15.4g [6] amendment. The revised version of the standard incorporates new modulation schemes targeted at different application domains, such as the SUN (Smart Utility Networks) modulations, which includes SUN-FSK

The authors thank the Brazilian National Council for Scientific and Technological Development (CNPq), the Generalitat de Catalunya (SGR-60-2017) and the Spanish Ministry of Science, Innovation and Universities (SPOTS RTI2018-095438-A-I00) for their support. This project is co-financed by the European Union Regional Development Fund within the framework of the ERDF Operational Program of Catalonia 2014-2020 with a 50\% grant of total cost eligible (4M€).
(Frequency-Shift Keying), SUN-OQPSK (Offset Quadrature Phase-Shift Keying) and SUN-OFDM (Orthogonal FrequencyDivision Multiplexing). These modulations can operate in both the Sub-GHz (i.e., $868 \mathrm{MHz}$ and $915 \mathrm{MHz}$ ) and $2.4 \mathrm{GHz}$ ISM (Industrial, Scientific and Medical) bands, and support various configuration modes, allowing to trade data rate and occupied bandwidth while providing robust long range communications.

Given the raising interest in dependability for low-power wireless communications [7] and the possibilities offered by the IEEE 802.15.4g SUN modulations [8], in this paper we explore the concept of modulation diversity as a means to improve reliability of low-power wireless networks. In particular, we introduce different modulation selection strategies and evaluate them using the dataset presented in [9], where a network with 11 nodes using the three different SUN modulations was deployed in a real industrial environment. The results of our study show that adding modulation diversity to packet transmissions can improve the global PDR (Packet Delivery Ratio). In addition, the use of adaptive modulation selection strategies allows the nodes to to deal with link quality spatial and temporal variations, reducing the RNP (Required Number of Packet Transmissions). The results also show that the more retransmissions per data packet are allowed the higher is the advantage of using the proposed adaptive modulation diversity strategies. To our knowledge, this is the first paper to propose and evaluate adaptive modulation selection for low-power wireless communications based on IEEE 802.15.4, and show how that it can improve PDR and RNP to meet the reliability level required by industrial applications.

The remaining of the paper is organized as follows. Section II introduces IEEE 802.15.4g SUN and modulation diversity, and presents the work related to both concepts. Section III proposes various strategies to implement modulation diversity for low-power wireless networks. Section IV provides an overview of the dataset and describes the methodology used to evaluate the proposed strategies to implement modulation diversity. Section V presents the results obtained using the real-world dataset. Finally, Section VI concludes the paper and outlines the future work. 


\section{BACKGROUND AND RELATED WORK}

In this section we present the background and the work related to IEEE $802.15 .4 \mathrm{~g}$ and modulation diversity.

\section{A. IEEE $802.15 .4 \mathrm{~g}$}

The IEEE 802.15.4g amendment [6] defines three new modulations targeted to SUN applications: SUN-FSK, SUNOQPSK and SUN-OFDM.

First, the SUN-FSK modulation is included mainly due to its power efficiency and to ensure compatibility with legacy systems. Three different operation modes are defined for each frequency band supported in the standard. They define modulation and channel parameters, such as the modulation type (BFSK or 4FSK), the channel spacing, and the modulation index. The data rate may vary from 50 to $200 \mathrm{kbps}$.

Second, the OQPSK modulation was introduced for the $2.4 \mathrm{GHz}$ band in the first version of the IEEE 802.15.4 standard, in 2003. It is combined with DSSS (Direct Sequence Spread Spectrum) and provides a data rate of $250 \mathrm{kbps}$ using $5 \mathrm{MHz}$ channels. In IEEE $802.15 .4 \mathrm{~g}$, OQPSK support is extended to other frequency bands, and is also combined with DSSS to provide data rates ranging from $6.25 \mathrm{kbps}$ to $500 \mathrm{kbps}$ depending on the spreading factor.

Finally, the SUN-OFDM modulation provides higher data rates and longer communication range, while dealing with interference and multi-path propagation. Various MCS (Modulation and Coding Schemes) are defined with different modulations (i.e., BPSK, QPSK, and 16-QAM), and frequency repetition configurations (i.e., 4x, 2x or no frequency repetition), allowing to provide data rates between $50 \mathrm{kbps}$ and $800 \mathrm{kbps}$.

Over the past few years several research papers have evaluated IEEE 802.15.4g networks, as summarized next.

In [10], the authors evaluate three IEEE 802.15.4g configurations operating at $2.4 \mathrm{GHz}$ : O-QPSK, OFDM with frequency repetition, and OFDM without frequency repetition. The authors show that channel hopping makes sense even when using SUN-OFDM, as the channel width is small and all sub-carriers are influenced in a similar way by multipath fading.

In [11], the authors evaluate the use of IEEE 802.15.4g for environmental observations. The results show that the longest radio links were obtained when using SUN-FSK or SUNOQSPK, since these radio settings have the highest sensitivity for the transceiver used in the experiments. In contrast, SUNOFDM provided lower PDR due to the lower output power allowed for this configuration.

In [12], the authors demonstrate the use of IEEE $802.15 .4 \mathrm{~g}$ for smart building applications using the $2.4 \mathrm{GHz}$ ISM band. The results show that for the considered indoor scenario, which is severely impacted by multi-path propagation, SUN-OFDM can provide better reliability than SUN-OQPSK.

In [8], the authors evaluate the interference robustness of the SUN-OFDM physical layer and compare it to the OQPSKDSSS physical layer using the SINR (Signal to Interference + Noise Ratio) as a metric. The results show that SUN-OFDM provides at least $6 \mathrm{~dB}$ protection regardless of interference type and packet length, while providing a higher spectral efficiency.
In [13] the effect of the WLAN interferers on the SUN-FSK physical layer at $2.4 \mathrm{GHz}$ band is investigated. The authors propose to minimize the impact of the WLAN interferer, by predicting its activity, to enhance the communication links of SUN devices (e.g., by using adaptive modulation or adaptive time shifting).

Finally, in [14], the authors propose a co-existence mechanism to mitigate the influence of inter-PHY interference in co-located IEEE 802.15.4g networks. By means of using a management mechanism to schedule mutual detection, using a common signaling mode for IEEE $802.15 .4 \mathrm{~g}$ networks with different physical layers in the same environment.

Overall, the related work presented in this subsection focuses on evaluating the suitability of the different modulations defined in the IEEE $802.15 .4 \mathrm{~g}$ standard for different scenarios. However, the authors do not propose or evaluate any mechanism to deal with the different challenges of lowpower wireless communications presented earlier. Hence, this lack of adaptability is the main driver of our research and the reason why we introduce the concept of modulation diversity.

\section{B. Modulation diversity}

Like other diversity schemes (i.e., time, frequency, space or polarization), modulation diversity is a method to improve the reliability of communications by using different modulations. That is, consecutive packets can be transmitted using two or more modulations (e.g., FSK or PSK), taking advantage of their different properties regarding propagation and interference effects. For example, it is well known that narrowband modulations, such as FSK, are more robust against interference, whereas wideband modulations, such as OQPSKDSSS, provide better tolerance against multi-path propagation.

Despite not being novel, using modulation diversity for low-power wireless communications has been limited due to the lack of standards and the unavailability of commercial transceivers supporting multiple modulations. However, with the emergence of the IEEE 802.15.4g standard and the recent availability of off-the-shelf transceivers that implement all defined modulations, the use of modulation diversity may now be explored to deal with the aforementioned challenges.

To implement modulation diversity, different strategies can be adopted depending on the side that determines which modulation scheme to use for a given packet. For example, the decision can be made at the transmitting node, using metrics that can be calculated on its behalf, such as the ARR (ACK Reception Ratio). Another possibility is to perform the decision at the gateway side, which may use physical layer related metrics (e.g., RSSI or LQI) or upper layer related metrics (e.g., PDR or number of duplicate packets) to estimate the quality of each link and decide when a node has to use another modulation configuration.

Over the last years, various authors have have proposed the use of modulation diversity for low-power wireless networks.

In [15], a dual mode IEEE 802.15.4 receiver is proposed. The receiver switches between two demodulator chains without changing the modulation scheme used by the transmitter. 
The receiver can choose between a MSK (Minimum Shift Keying) detector or a OQPSK detector to trade energy consumption, latency, and reliability. In addition, the receiver can define the mode based on a SNR indicator, to optimize performance. However, the authors do not consider the use of the SUN modulations, neither propose the use of different modulations to transmit the packets.

In [16] the authors propose using cooperative modulation diversity to improve reliability. In particular, nodes rotate a QPSK constellation and interleave the phase and quadrature components independently. However, this approach requires modification at the physical layer, and the use of relay nodes, which can be difficult in sparse mesh networks or networks with star topology.

In summary, despite these papers propose the use of modulation diversity, to the best of our knowledge this is the first paper to propose and evaluate an adaptive modulation selection strategies for low-power wireless networks, which may have an impact to the increase of reliability while reducing energy consumption. Hence, the remainder of the paper focuses on proposing and evaluating various adaptive modulation selection strategies.

\section{Adaptive Modulation Diversity Strategies}

Based on the definition of modulation diversity introduced earlier, in this section we present three adaptive modulation selection strategies (named $I M, 2 M$ and $3 M$, respectively) aimed at improving link reliability of IEEE $802.15 .4 \mathrm{~g}$ networks.

To implement these policies, in this paper we use the ARR (ACK Reception Ratio) as the link quality metric. The ARR is defined as the ratio between the number of ACKs received successfully and the number of transmitted packets in a given interval. That is, nodes store the number of transmissions and the number of ACKs received successfully for each SUN modulation, and a new ARR value is calculated after the transmission of $N$ packets using each SUN modulation.

The first strategy, referred as $1 M$, only uses one IEEE 802.15.4g SUN modulation (i.e., SUN-FSK, SUNOQPSK or SUN-OFDM) to allow nodes to transmit packets, and a switch between modulations occurs only when the link quality drops below a given threshold $t$. That is, if the modulation being used has a link quality above the given threshold the modulation is used in the next transmission. Otherwise, the next modulation is selected for the coming transmissions according to the following order: SUN-FSK, SUN-OQPSK, and SUN-OFDM. Thus, using this strategy the node may perform all transmission attempts using the same modulation, unless a switch occurs in the middle of a sequence of attempts.

The second strategy, named $2 M$, uses two of the three IEEE $802.15 .4 \mathrm{~g}$ SUN modulations to allow nodes to transmit packets. These modulations are named $m_{1}$ and $m_{2}$ respectively, and are used in a round-robin fashion for each packet transmission. However, when the link quality for one of the modulations being used drops below a given threshold $t$, that modulation is switched to the third modulation that is currently not being used. When this occurs, the modulation that remains is redefined as $m_{1}$, and the new modulation to be used is defined as $m_{2}$. For example, modulation $m_{1}$ is SUN-FSK and is used in odd attempts, while modulation $m_{2}$ is SUN-OQPSK and is used in even attempts.

The third and final strategy, named $3 M$, enforces the use all three IEEE 802.15.4g SUN modulations to allow nodes to transmit packets. Before transmitting a packet, each node selects one modulation based on their probability of use, which is determined according to the following equation:

$$
p_{i}=\frac{\left(1+a_{i}\right)^{w}}{\sum_{k=0}^{k<3}\left(1+a_{k}\right)^{w}},
$$

in which $p_{i}$ is the probability of using modulation $i$ (where 0 is SUN-FSK, 1 is SUN-OQPSK, and 2 is SUN-OFDM), $a_{i}$ is the ARR for modulation $i$, and $w$ is a weight factor that is used to control the differences between the calculated probabilities. However, if a given transmission attempt fails the packet retransmission cannot use the same modulation. Instead, the modulation used is selected from the remaining modulations according to its respective probability, thus allowing to increase diversity. It is important to remark that these probabilities are computed periodically based on the quality of the links, and every time a new link quality is computed for one of the modulations, the probabilities of the other modulations are also updated. Note that the ARR for a given modulation is calculated based only on the packets transmitted using this specific modulation. Thus, when a modulation has a high $p$, its ARR is updated more frequently. Hence, if the link quality for a given modulation that has a high $p$ drops, the probabilities will be adjusted fast. However, if a modulation with a low $p$ starts to present good quality, it will take more time until the probabilities are adjusted.

\section{Evaluation Methodology}

In this section we introduce the metrics, the dataset, the simulator and the parameters that we use to evaluate the adaptive modulation diversity strategies presented earlier.

\section{A. Performance metrics}

The metrics used to evaluate the performance of the proposed adaptive modulation diversity strategies are the PDR (Packet Delivery Ratio) and the RNP (Required Number of Packet Transmissions) [17]. The PDR represents the ratio between received and transmitted packets at the application layer, whereas the RNP represents the average number of packet repetitions before a successful packet reception at the application layer. Please notice that the RNP metric is complementary to the PDR to evaluate link quality. Despite being influenced by the associated PDR, lower values of RNP indicate that most of the packets were delivered in the first transmission attempt. 


\section{B. Dataset overview}

To evaluate the proposed modulation diversity strategies we use the dataset provided in [9], where the authors provide a detailed description of the experiment and a general statistical analysis of various metrics (i.e., RSSI and PDR).

As a summary, the connectivity traces have been obtained from a network of 11 OpenMote-B [18] nodes deployed for 99 days in an industrial environment (i.e., a clothing warehouse). The sensor network uses a star-topology and the nodes have been located at distances ranging from $34 \mathrm{~m}$ to $274 \mathrm{~m}$ with respect to the gateway. The gateway contains three independent IEEE $802.15 .4 \mathrm{~g}$ SUN radio transceivers to be able to receive packets simultaneously for all three modulations. Every minute each node generates a data packet with sensor data, which is transmitted 3 times with each IEEE 802.15.4g SUN modulation. All modulations provide an effective data rate of $50 \mathrm{kbps}$ and CSMA/CA is used for channel access to avoid collisions. Considering the total of nodes and packets transmitted, the overall channel occupation is $0.85 \%$ (i.e., $506.88 \mathrm{~ms}$ per minute). More details about the evaluated modulations are described in [9].

As shown in [9], during the experiment the overall PDR varied between $69.9 \%$ and $89.8 \%$ at the physical layer depending on the modulation. However, thanks to packet replication (i.e., 9 transmissions per data packet), the overall PDR at the application layer was $100.0 \%$. But despite meeting the application requirements, the high number of transmissions increases node energy consumption and overloads the network, limiting its durability and scalability.

Table I shows the PDR and RNP values for each modulation combination using two transmission attempts with two possible orders (direct and reverse order). As it can be observed, each node presents different levels of reliability for the different configurations but, in general, we observe that the overall PDR is similar in all configurations regardless of the order they are used. In contrast, we may observe significant differences in RNP values. For example, for the configuration using SUN-FSK and SUN-OFDM, when using SUN-OFDM in the first attempt (reverse order), the RNP is $15 \%$ higher. This can be explained by the fact that SUN-FSK presented the highest PDR during most of the time for most nodes. Thus, the packets are delivered in the first attempt more frequently when using the direct order.

Table II shows the percentage of time that each modulation provides the best PDR at the physical layer for all nodes. For this analysis, the PDR is calculated every 5 minutes, during which each node transmits 15 packets using each modulation. Since nodes transmit at fixed intervals and the number of transmissions is known a priori, it is possible to determine the PDR for all modulations, even when no packet is received for a specific modulation during that interval. From the results we observe that, although SUN-FSK is the best modulation for most nodes, for nodes 56-53,55-AD, 55-E4, and 56-0B, the SUN-OFDM provided the best PDR at the physical layer during more than $15 \%$ of the time. In contrast, SUN-OQPSK provided the best PDR during more than $15 \%$ of the time for nodes 56-53, 55-E4, 55-99, and 55-65.

Hence, we can conclude that no single modulation is capable of providing the best PDR during all the time, which confirms the need of the adaptive modulation selection strategies presented in Section III.

\section{Simulation setup}

To evaluate the proposed modulation diversity strategies we have developed a Python simulator ${ }^{1}$, that uses the PDR traces from the dataset presented in Section IV-B.

The simulator uses the dataset to compute the physical layer PDR for all modulations and nodes for each 5 minute interval. Then, based on the PDR, the simulator determines if each transmitted packet and its acknowledgement is successfully delivered or not. When an acknowledgement packet is not received, due to a failure in one of the two transmissions (packet or ACK), the node may retransmit the same packet, according to the number of allowed retries.

It is important to mention that, since in the dataset packets are transmitted without acknowledgements, in the simulations the calculated values of PDR are also used to determine the PDR of the transmissions of the ACK packets. Thus, the following assumptions are made: (1) the PDR of the two directions of the links are equal, meaning that links are symmetric; (2) the additional packets generated due to the use of acknowledgement do not cause significant influence on the PDR. As discussed before, the channel occupation is very low. Thus, we do not expect a high impact in performance caused by self-interference, even with the additional acknowledgement packets needed by the adaptive modulation selection strategies. In addition, by using acknowledgement the number of retransmissions can be reduced, since no blind retransmissions are made.

Finally, in addition to the modulation selection strategies presented in Section III, we have also implemented three complementary strategies for comparison purposes: roundrobin, random and best. In the round-robin strategy the nodes use all three modulations in a round-robin fashion. In the random strategy the nodes select the modulation to transmit a packet randomly with equal probabilities for each one. Finally, in the best strategy we simulate the PDR achieved if the nodes always use the best modulation during all the time. Hence, the round-robin and random represent two strategies with expected average performance, whereas the best strategy represents an upper bound given the channel conditions.

\section{Evaluation parameters}

To evaluate the adaptive modulation diversity policies presented in Section III we use the following parameters:

- $t$, which is the threshold that we use to determine if the modulation has to be changed. In this paper we use $t=$ 0.9 , since a PDR of $90 \%$ at the physical layer provides an

\footnotetext{
${ }^{1}$ The source code and the trace files can be found in a public GitHub repository: https://github.com/GComPI-IFPB/IEEE802154g-adaptive-modulation
} 


\begin{tabular}{|c|c|c|c|c|c|c|c|c|c|c|c|c|}
\hline \multirow{2}{*}{$\begin{array}{c}\text { EUI-64 } \\
\text { (last 2-bytes) }\end{array}$} & \multicolumn{2}{|c|}{ SUN-FSK } & \multicolumn{2}{|c|}{ SUN-OQPSK } & \multicolumn{2}{|c|}{ SUN-OFDM } & \multicolumn{2}{|c|}{$\begin{array}{l}\text { SUN-FSK and SUN-OQPSK } \\
\text { (reverse order) }\end{array}$} & \multicolumn{2}{|c|}{$\begin{array}{l}\text { SUN-FSK and SUN-OFDM } \\
\text { (reverse order) }\end{array}$} & \multicolumn{2}{|c|}{$\begin{array}{l}\text { SUN-OQPSK and SUN-OFDM } \\
\text { (reverse order) }\end{array}$} \\
\hline & PDR (\%) & RNP & PDR (\%) & RNP & PDR (\%) & RNP & PDR (\%) & RNP & PDR (\%) & RNP & PDR (\%) & RNP \\
\hline $56-53$ & 82.5 & 1.59 & 86.3 & 1.50 & 87.0 & 1.49 & $86.9(86.0)$ & $1.51(1.52)$ & $87.0(88.3)$ & $1.50(1.51)$ & $88.6(\mathbf{9 0 . 3})$ & $1.45(1.44)$ \\
\hline $55-\mathrm{AD}$ & 87.6 & 1.35 & 86.5 & 1.40 & 95.0 & 1.22 & $87.5(86.9)$ & $1.33(1.43)$ & $92.2(94.6)$ & $1.25(1.30)$ & $91.4(94.4)$ & $1.33(1.22)$ \\
\hline $55-\mathrm{E} 4$ & 81.4 & 1.53 & 84.1 & 1.46 & 80.1 & 1.63 & $87.3(86.1)$ & $1.42(1.44)$ & $86.2(84.6)$ & $1.43(1.59)$ & $86.5(85.8)$ & $1.41(1.53)$ \\
\hline $55-99$ & 89.0 & 1.33 & 88.1 & 1.36 & 80.6 & 1.63 & $89.7(88.3)$ & $1.32(1.36)$ & $89.6(89.0)$ & $1.32(1.49)$ & $88.5(89.0)$ & $1.36(1.48)$ \\
\hline $55-\mathrm{DD}$ & 93.4 & 1.17 & 93.3 & 1.19 & 86.6 & 1.39 & 94.0 (93.9) & $1.16(1.19)$ & $94.0(93.9)$ & $1.16(1.19)$ & $93.8(93.9)$ & $1.18(1.29)$ \\
\hline $55-65$ & 92.0 & 1.42 & 96.4 & 1.23 & 90.4 & 1.50 & $96.6(\mathbf{9 6 . 8})$ & $1.35(1.23)$ & $94.2(93.1)$ & $1.39(1.46)$ & $96.2(96.1)$ & $1.23(1.41)$ \\
\hline $56-0 \mathrm{~B}$ & 83.0 & 1.58 & 83.2 & 1.58 & 77.6 & 1.78 & $83.6(83.4)$ & $1.56(1.58)$ & $83.8(87.0)$ & $1.55(1.60)$ & $84.1(86.8)$ & $1.58(1.58)$ \\
\hline $56-32$ & 98.3 & 1.08 & 95.2 & 1.19 & 87.9 & 1.37 & $98.4(97.7)$ & $1.07(1.16)$ & 97.9 (97.9) & $1.06(1.29)$ & $95.9(96.5)$ & $1.15(1.28)$ \\
\hline $55-\mathrm{B} 3$ & 97.6 & 1.09 & 98.5 & 1.15 & 92.1 & 1.42 & $99.0(98.8)$ & $1.07(1.12)$ & $98.5(98.1)$ & $1.06(1.37)$ & $98.3(97.9)$ & $1.15(1.36)$ \\
\hline $55-63$ & 92.6 & 1.29 & 86.7 & 1.47 & 86.4 & 1.50 & $91.0(\mathbf{9 3 . 3})$ & $1.29(1.46)$ & 85.4 (91.6) & $1.35(1.69)$ & $82.8(86.3)$ & $1.52(1.68)$ \\
\hline $63-0 \mathrm{~A}$ & 94.9 & 1.17 & 94.0 & 1.22 & 54.8 & 2.89 & 95.5 (95.6) & $1.15(1.21)$ & $92.8(93.0)$ & $1.19(1.82)$ & $90.2(90.2)$ & $1.25(1.86)$ \\
\hline Overall & 90.2 & 1.33 & 90.2 & 1.34 & 83.5 & 1.62 & $91.8(91.5)$ & $1.29(1.34)$ & 91.1 (91.9) & $1.30(1.49)$ & $90.6(91.6)$ & $1.33(1.47)$ \\
\hline Maximum & 98.3 & 1.59 & 98.5 & 1.58 & 95.0 & 2.89 & $99.0(98.8)$ & $1.56(1.58)$ & $98.5(98.1)$ & $1.56(1.82)$ & $98.3(97.9)$ & $1.58(1.86)$ \\
\hline Minimum & 81.4 & 1.08 & 83.2 & 1.15 & 54.8 & 1.22 & 83.6 (83.4) & $1.07(1.12)$ & $83.8(84.6)$ & $1.06(1.29)$ & $82.8(85.8)$ & $1.15(1.23)$ \\
\hline
\end{tabular}

PDR AND RNP VALUES FOR EACH CONFIGURATION USING 2 TRANSMISSION ATTEMPTS.

\begin{tabular}{|c|c|c|c|c|c|}
\hline EUI-64 & Distance & Height & SUN-FSK & SUN-OQPSK & SUN-OFDM \\
\hline $56-53$ & 34.0 & 12.0 & 49.24 & 29.99 & 20.75 \\
\hline $55-\mathrm{AD}$ & 63.0 & 2.0 & 59.58 & 9.76 & 30.65 \\
\hline $55-\mathrm{E} 4$ & 80.0 & 6.0 & 37.6 & 21.44 & 40.94 \\
\hline $55-99$ & 115.1 & 2.0 & 71.65 & 19.83 & 8.5 \\
\hline $55-\mathrm{DD}$ & 115.1 & 6.0 & 85.97 & 8.01 & 6.0 \\
\hline $55-65$ & 115.1 & 10.0 & 67.0 & 28.79 & 4.19 \\
\hline $56-0 \mathrm{~B}$ & 115.1 & 14.0 & 70.87 & 13.29 & 15.83 \\
\hline $56-32$ & 172.5 & 2.0 & 94.28 & 3.01 & 2.69 \\
\hline $55-\mathrm{B} 3$ & 221.4 & 2.0 & 80.03 & 10.74 & 9.21 \\
\hline $55-63$ & 224.4 & 2.0 & 90.82 & 8.84 & 0.33 \\
\hline $63-0 \mathrm{~A}$ & 273.5 & 2.0 & 86.11 & 11.76 & 2.12 \\
\hline
\end{tabular}

PERCENTAGE OF TIME FOR WHICH EACH MODULATION PROVIDES THE BEST PDR (PACKET DELIVERY RATIO).

average PDR at the application layer of $99 \%$ and $99.9 \%$ when using 2 and 3 transmission attempts, respectively.

- $N$, which is the number of packets after which we recompute the ARR values. If the value of $N$ is low, the estimator becomes unstable, since a single transmission failure causes a significant reduction in the ARR, triggering the modulation switch procedure in the strategies. Contrarily, if $N$ is high the estimator becomes less responsive to modifications in link quality. Hence, in this paper we use $N=10$, requiring at least two transmissions failures inside an ARR window to drop below the threshold (i.e., ARR $\leq 0.8)$, since we use $t=0.9$.

- $w$, which is the weight factor used to control the differences between the calculated probabilities. In this particular case we use $w=20$. Figure 1 shows the influence of $w$ in the value of $\frac{\mathrm{RNP}}{\mathrm{PDR}}$. It is possible to see that the curve stabilizes from $w=20$, considering two transmission attempts. In future work the dynamic adjustment of this factor will be explored.

\section{RESUlTS}

In this section we present and discuss the results obtained with the proposed adaptive modulation diversity strategies (i.e., $1 M, 2 M, 3 M$, random, round-robin and best).

To obtain these results we have performed ten repetitions of each experiment, as explained earlier, and calculated the mean and standard values. In addition, to better analyse the results,

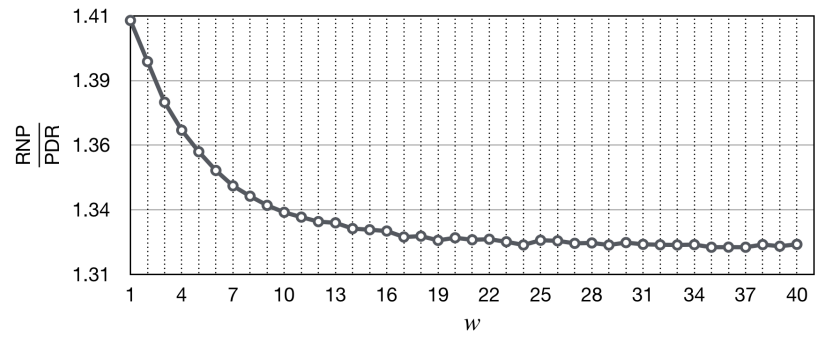

Fig. 1. Influence of $w$ in the $3 \mathrm{M}$ strategy.

we have created clusters based on the distance $(d)$ between nodes and the gateway, allowing to make comparisons within similar groups. In particular, we have defined three groups of nodes: (1) close (i.e., $d<80 \mathrm{~m}: 56-53,55-\mathrm{AD}$ and 55-E4), (2) medium (i.e., $80 \leq d<150 \mathrm{~m}$ : 55-99, 55-DD, 55-65 and 56-0B) and, (3) far (i.e., $d \geq 150 \mathrm{~m}: 56-32,55-\mathrm{B} 3$, $55-63$ and 63-0A).

Figures 2, 3 and 4 show the PDR using up to two transmission attempts per packet, for the nodes of the close, medium, and far groups, respectively. From the graphs it is possible to notice that OFDM only provided a high PDR for the close group. This can be explained by the fact that the SUNOFDM transmit power is $6 \mathrm{~dB}$ lower than SUN-FSK and SUNOQPSK, and its sensitivity is $3 \mathrm{~dB}$ and $4 \mathrm{~dB}$ lower than these modulations.

Besides, the global PDR when using the proposed diversity strategies is slightly better when compared to the other scenarios, and with a higher minimum value. In particular, the $2 M$ and $3 M$ configurations provide the best global PDR. This occurred because the $2 M$ and $3 M$ strategies present a higher level of diversity when compared to $l M$. Thus, the probability of using at least one good modulation for every packet in one of the attempts is higher for these strategies.

Finally, the diversity strategies presented a worst result only when one of the modulations was much better than the others (i.e., nodes 55-AD, 55-65, and 55-63). In cases where at least two modulations presented similar PDR, the diversity strategies provided a better performance in comparison to the 
use of only one modulation.

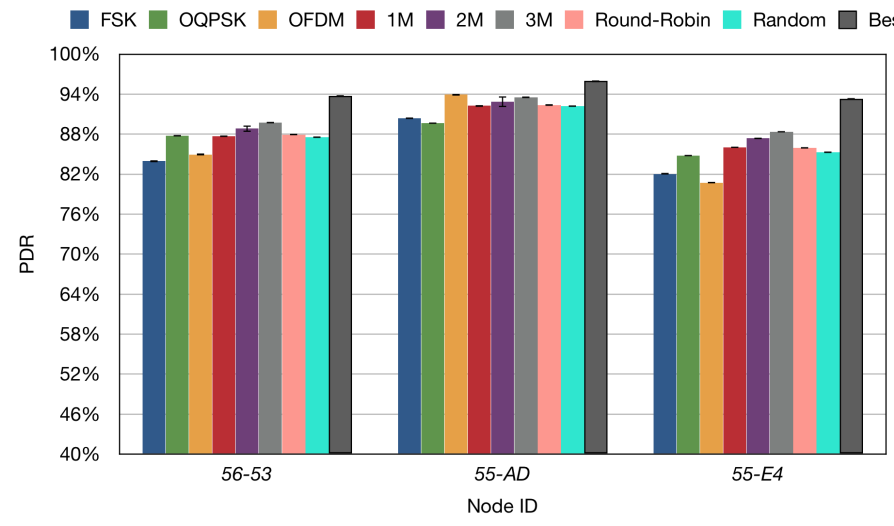

Fig. 2. PDR of the nodes that are close to the gateway. Bars represent the mean and error bars represent the standard deviation.

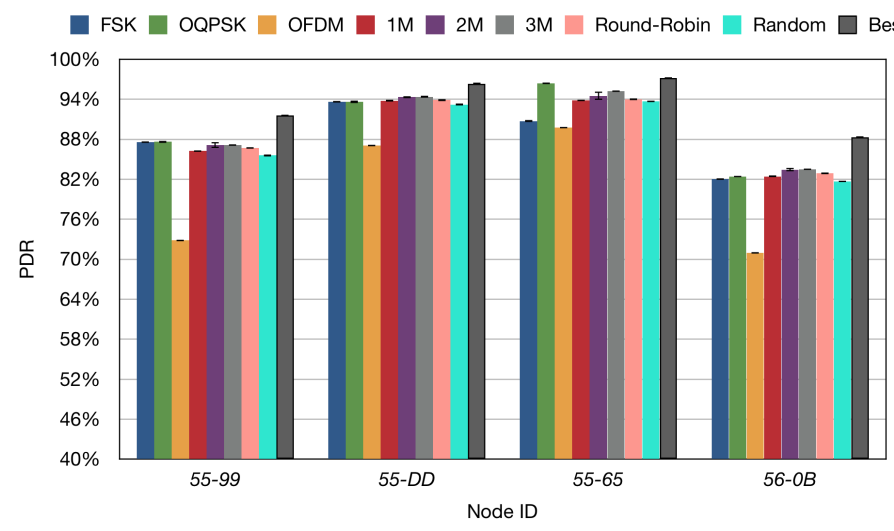

Fig. 3. PDR of the nodes that are at medium distance from the gateway. Bars represent the mean and error bars represent the standard deviation.

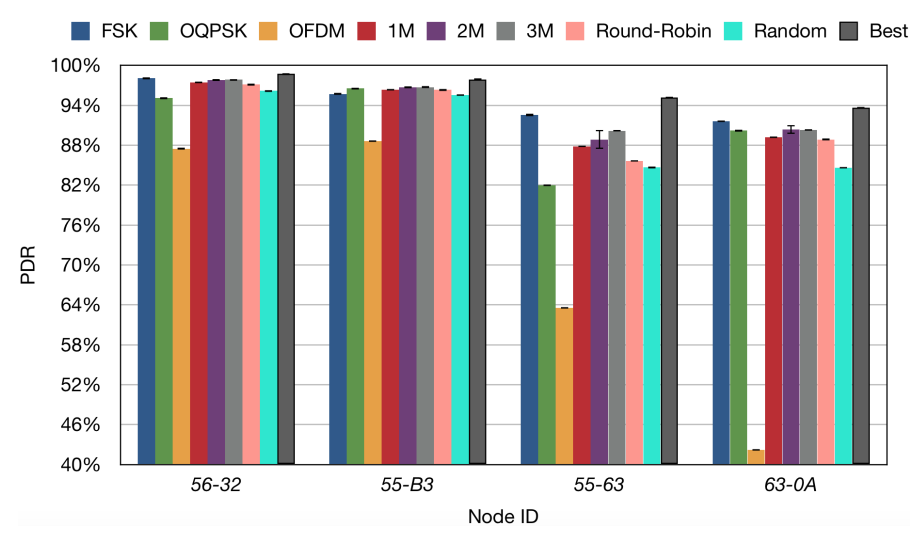

Fig. 4. PDR of the nodes that are at far from the gateway. Bars represent the mean and error bars represent the standard deviation.

Now we focus on the performance of the modulation selection strategies when more than two packet transmissions are allowed. Please notice that with more packet transmissions it is possible to increase the PDR at the application layer, but it also increases network traffic and energy consumption.
Thus, besides PDR, in this section we also analyze the RNP, to evaluate the average number of transmissions attempts before a packet reception for each evaluated configuration.

As shown in Figure 5, the more retransmissions per data packet are allowed the higher is the advantage of using the proposed adaptive modulation selection strategies compared to using a single modulation. Similarly, the more retransmissions per data packet the lower is the difference between the PDR achieved with the proposed adaptive modulation selection strategies and the PDR achieved in the best scenario. For example, the overall PDR obtained using up to two transmission attempts is equal to $89.8 \%, 91.5 \%$, and $94.7 \%$, for the SUNFSK (the best single-modulation configuration), $3 M$, and best configurations, respectively, while the overall PDR is equal to $95.2 \%, 97.3 \%$ and $98.5 \%$ when using up to 6 transmission attempts. At the same time, the RNP for the SUN-FSK, and $3 M$ configurations increase $41.6 \%$, and $34.5 \%$, respectively, when the number of attempts increases from two to six. Thus, by using the $3 M$ strategy it is possible to achieve a higher absolute increase in PDR, with a lower increase in RNP, when more retries are allowed.

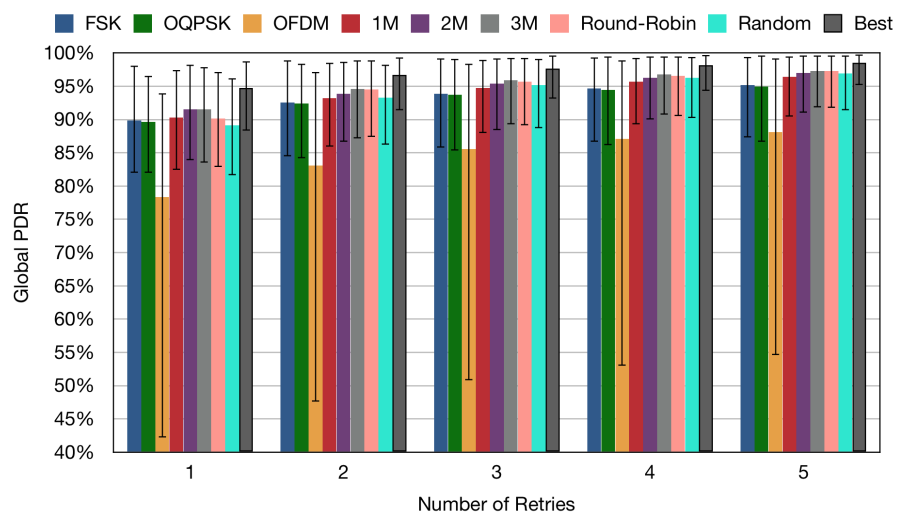

Fig. 5. Global PDR for the different adaptive modulation diversity strategies depending on the number of retries. Bars represent the mean and error bars represent the minimum and maximum PDR for a specific node.

Figure 6 shows the relation between PDR and RNP for the different adaptive modulations strategies presented in the paper, as well as for SUN-FSK, which is the single-modulation configuration that offers the best performance. Each line color represents a given modulation diversity strategy and each point the number of packet retransmissions allowed for that strategy (i.e., 1 to 5). Here it is possible to notice that the use of modulation diversity indeed improves the reliability, mainly when more retransmissions are allowed. In particular, the PDR provided by the $3 M$ strategy when using up to two retransmissions is higher than the PDR achieved using only the SUN-FSK with up to three retransmissions (i.e., $94.6 \%$ compared to $93.8 \%$ ).

Despite the PDR of the different modulation diversity strategies are similar, specially when more retransmissions are allowed, the adaptive strategies (i.e. $1 M, 2 M$, and $3 M$ ) present lower RNP levels in comparison to the round-robin and random strategies. In particular, for the scenario with up 


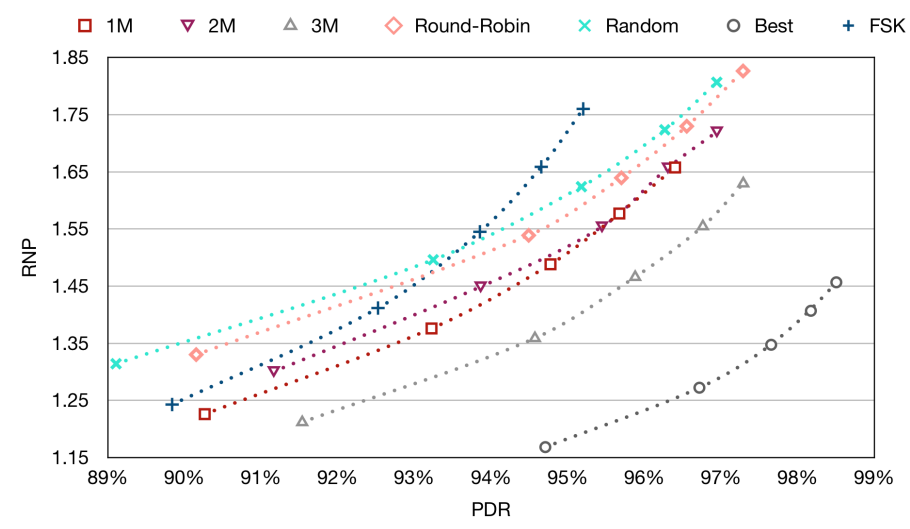

Fig. 6. RNP vs PDR for the different adaptive modulation diversity strategies. Each point represents the number of allowed packet retransmissions allowed for that parciular strategy (i.e., from 1 to 5).

to two transmissions attempts, the $3 M$ presented a higher PDR compared to round-robin (i.e., $91.5 \%$ compared to $90.1 \%$ ), and a RNP 9.8\% lower. For the scenario with up to six transmission attempts, the $3 M$ and round-robin strategies presented the highest PDR (i.e., $97.3 \%$ for both), but the RNP of the $3 M$ strategy was $12.1 \%$ lower. Hence, we confirm that implementing modulation diversity strategies is a useful tool to improve link reliability for IEEE $802.15 .4 \mathrm{~g}$ networks.

\section{CONCLUSiOnS AND Future WORK}

In this paper we have proposed and evaluated different adaptive modulation selection strategies for IEEE $802.15 .4 \mathrm{~g}$ SUN networks. According to the simulation results with a realworld dataset, using any modulation diversity strategy allows to increase the PDR (Packet Delivery Ratio) with respect to using a single modulation. Furthermore, using the proposed modulation diversity strategies (i.e., $1 M, 2 M$ and $3 M$ ) allows to further increase PDR with respect to the random and roundrobin strategies, but leads to a lower RNP (Required Number of Packet Transmissions) regardless of the number of packet retransmissions. In particular, the round-robin and $3 M$ strategies present the highest PDR among all modulation selection strategies, but $3 M$ provides a slightly better overall PDR and a lower RNP (i.e., a PDR up to $1.4 \%$ higher, and a RNP between $9.8 \%$ and $12.1 \%$ lower, depending on the number of allowed packet retransmissions). Overall, this leads to less packet retransmissions regardless of context which, in turn, helps reducing energy consumption and network congestion.

Given this promising results, as future work we will explore hybrid modulation selection strategies, which allow to modify both the number retransmissions and the modulations based on the relative link quality. Other strategies for link quality estimation will also be investigated to identify and select the best modulation in an accurate and responsive way.

\section{REFERENCES}

[1] D. V. Queiroz, M. S. Alencar, R. D. Gomes, I. E. Fonseca, and C. Benavente-Peces, "Survey and systematic mapping of industrial Wireless Sensor Networks," Journal of Network and Computer
Applications, vol. 97, no. August, pp. 96-125, 2017. [Online]. Available: http://dx.doi.org/10.1016/j.jnca.2017.08.019

[2] E. Callaway, P. Gorday, L. Hester, J. A. Gutierrez, M. Naeve, B. Heile, and V. Bahl, "Home networking with ieee 802.15.4: a developing standard for low-rate wireless personal area networks," IEEE Соттиnications Magazine, vol. 40, no. 8, pp. 70-77, Aug 2002.

[3] X. Vilajosana, T. Watteyne, T. Chang, M. Vučinić, S. Duquennoy, and P. Thubert, "IETF 6TiSCH: A Tutorial," IEEE Communications Surveys Tutorials, pp. 1-1, 2019.

[4] J. d. Armas, P. Tuset, T. Chang, F. Adelantado, T. Watteyne, and $\mathrm{X}$. Vilajosana, "Determinism through path diversity: Why packet replication makes sense," in 2016 International Conference on Intelligent Networking and Collaborative Systems (INCoS), Sep. 2016, pp. 150154.

[5] "IEEE Standard for Low-Rate Wireless Networks," IEEE Std 802.15.42015 (Revision of IEEE Std 802.15.4-2011), pp. 1-709, April 2016.

[6] "IEEE Standard for Local and metropolitan area networks-Part 15.4: Low-Rate Wireless Personal Area Networks (LR-WPANs) Amendment 3: Physical Layer (PHY) Specifications for Low-Data-Rate, Wireless, Smart Metering Utility Networks," IEEE Std 802.15.4g-2012 (Amendment to IEEE Std 802.15.4-2011), pp. 1-252, April 2012.

[7] P. Thubert, D. Cavalcanti, X. Vilajosana, and C. Schmitt, "Reliable and available wireless technologies," Working Draft, IETF Secretariat, Internet-Draft draft-thubert-rawtechnologies-04, January 2020, http://www.ietf.org/internet-drafts/ draft-thubert-raw-technologies-04.txt. [Online]. Available: http: //www.ietf.org/internet-drafts/draft-thubert-raw-technologies-04.txt

[8] P. Tuset-Peiró, F. Vázquez-Gallego, J. Muñoz, T. Watteyne, J. AlonsoZarate, and X. Vilajosana, "Experimental Interference Robustness Evaluation of IEEE 802.15.4-2015 OQPSK-DSSS and SUN-OFDM Physical Layers for Industrial Communications," Electronics, vol. 8, no. 9, p. 1045, Sep 2019. [Online]. Available: http://dx.doi.org/10.3390/ electronics 8091045

[9] P. Tuset-Peiró, R. D. Gomes, P. Thubert, and X. Vilajosana, "Evaluating IEEE 802.15.4g SUN for Dependable Low-Power Wireless Communications In Industrial Scenarios," Sensors (available on Preprints), vol. XX, no. XX, 2020.

[10] J. Muñoz, P. Muhlethaler, X. Vilajosana, and T. Watteyne, "Why channel hopping makes sense, even with ieee802.15.4 ofdm at 2.4 ghz," in 2018 Global Internet of Things Summit (GIoTS), June 2018, pp. 1-7.

[11] J. Muñoz, T. Chang, X. Vilajosana, and T. Watteyne, "Evaluation of ieee802.15.4g for environmental observations," Sensors, vol. 18, no. 10, 2018. [Online]. Available: https://www.mdpi.com/1424-8220/ $18 / 10 / 3468$

[12] J. Muñoz, E. Riou, X. Vilajosana, P. Muhlethaler, and T. Watteyne, "Overview of ieee802.15.4g ofdm and its applicability to smart building applications," in 2018 Wireless Days (WD), April 2018, pp. 123-130.

[13] A. Gouissem, R. Hamila, N. Al-Dhahir, L. Ben-Brahim, and A. Gastli, "Time-dependent bit error rate analysis for smart utility networks in the presence of wlan interferers," IEEE Systems Journal, pp. 1-11, 2019.

[14] C. Sum, F. Kojima, and H. Harada, "Performance analysis of a multiphy coexistence mechanism for ieee 802.15.4g fsk network," in 2013 IEEE Wireless Communications and Networking Conference (WCNC), April 2013, pp. 41-46.

[15] M. A. Zubair, A. K. Nain, J. Bandaru, P. Rajalakshmi, and U. B. Desai, "Reconfigurable dual mode ieee 802.15.4 digital baseband receiver for diverse iot applications," in 2016 IEEE 3rd World Forum on Internet of Things (WF-IoT), Dec 2016, pp. 389-394.

[16] M. P. Sousa, W. T. A. Lopes, F. Madeiro, and M. S. Alencar, "Cooperative modulation diversity applied to heterogeneous wireless sensor networks," in 2014 IEEE International Conference on Distributed Computing in Sensor Systems, May 2014, pp. 338-343.

[17] N. Baccour, A. Koubâa, L. Mottola, M. A. Zúñiga, H. Youssef, C. A. Boano, and M. Alves, "Radio link quality estimation in wireless sensor networks: A survey," ACM Transactions on Sensor Networks (TOSN), vol. 8, no. 4, p. 34, 2012.

[18] P. Tuset-Peiró, X. Vilajosana, and T. Watteyne, "OpenMote+: A RangeAgile Multi-Radio Mote," in Proceedings of the 2016 International Conference on Embedded Wireless Systems and Networks, ser. EWSN '16. USA: Junction Publishing, 2016, pp. 333-334. [Online]. Available: http://dl.acm.org/citation.cfm?id=2893711.2893794 